\title{
Subsurface charge accumulation imaging of a quantum Hall liquid
}

\section{S. H. Tessmer, P. I. Glicofridis, R. C. Ashoori, L. S. Levitov \& M. R. Melloch}

Nature 392, 51-54 (1998)

In this Letter in the 5 March issue, some of the superscript lettering on Figs 2-4 was printed incorrectly. In addition, the contrast was low in some copies of the journal. Reprints with the figures correctly produced are available, and they are reprinted here.
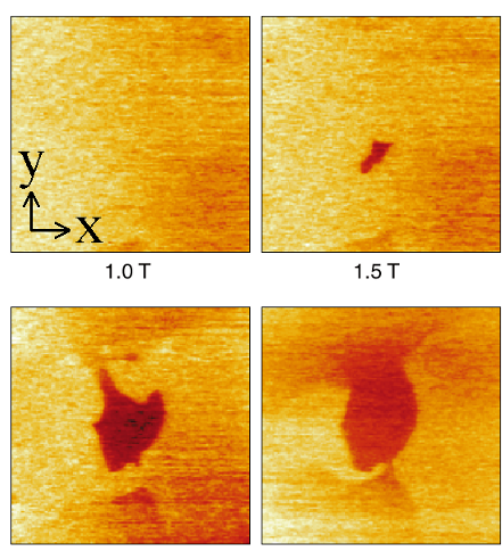

$3.0 \mathrm{~T}$

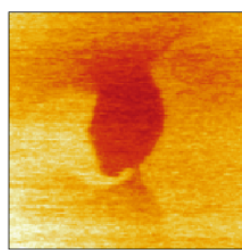

$3.8 \mathrm{~T}$
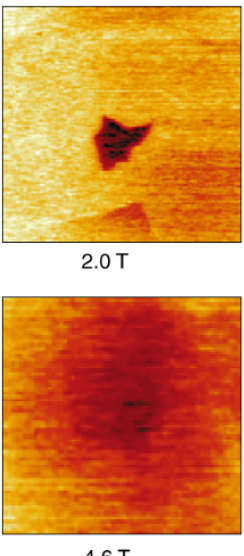

$4.6 \mathrm{~T}$

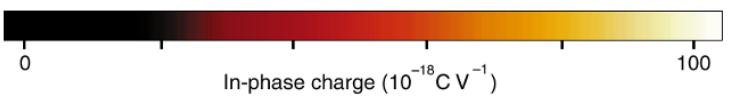

Figure 2

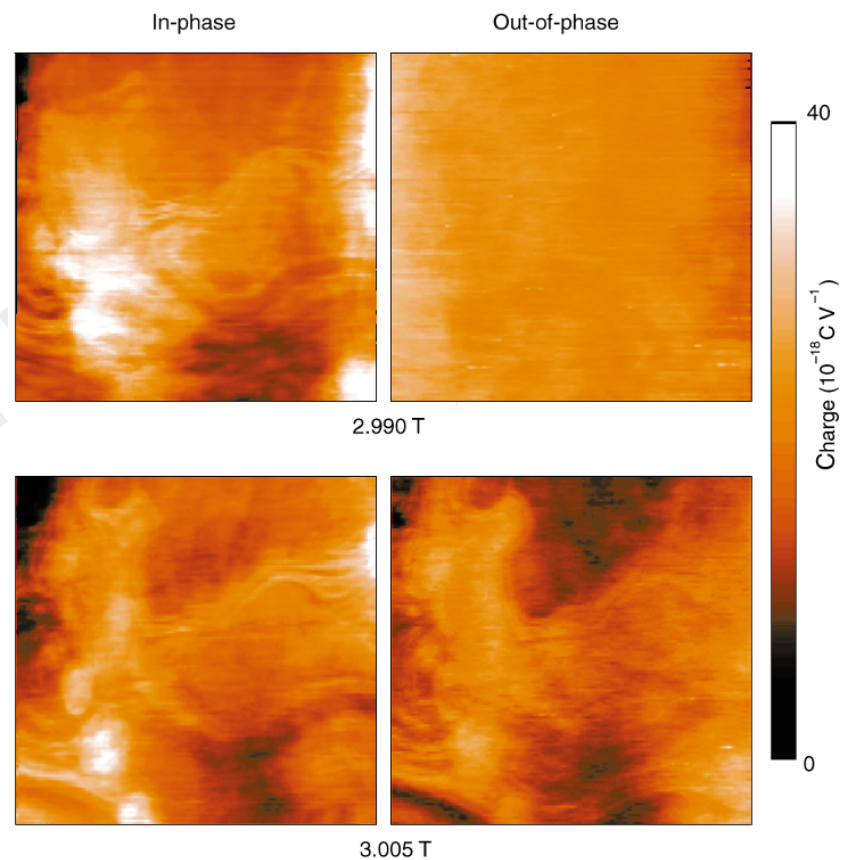

Figure 4
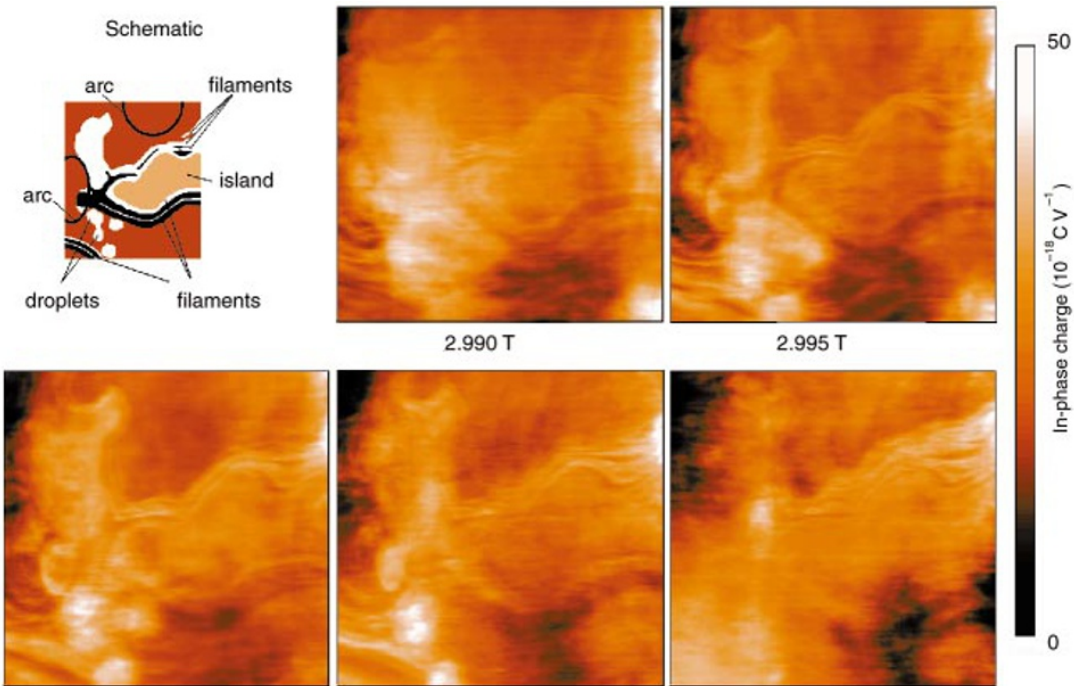

$3.000 \mathrm{~T}$

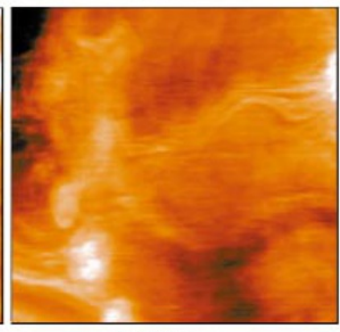

$2.995 \mathrm{~T}$

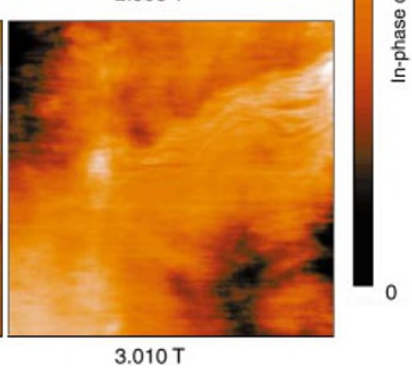


15. Bus, S. J., A'Hearn, M. F., Bowell, E. \& Stern, S. A. 2060 Chiron: evidence for activity near aphelion Icarus (submitted)

16. Degewij, J. \& Van Houten, C. J. in Asteroids (ed. Gehrels, T.) 417-435 (Univ. Arizona Press, Tucson, 1979).

17. Tody, D. in Astronomical Data Analysis Software and Systems II (eds Hanisch, R. J., Brissenden, R. J. V. \& Barnes, J.) 173 (ASP Conf. Ser. 52, Tucson, 1993).

Acknowledgements. We thank the NASA Origins of Solar Systems program for support of this work, and the Steward Observatory Telescope Allocation Committee for consistent allocation of telescope time.

Correspondence and requests for materials should be addressed to S.C.T. (e-mail address: tegler@proto.phy.nau.edu).

\section{Subsurface charge} accumulation imaging of a quantum Hall liquid

\section{S. H. Tessmer ${ }^{\star}$, P. I. Glicofridis ${ }^{\star}$, R. C. Ashoori ${ }^{\star}$, L. S. Levitov ${ }^{\star} \&$ M. R. Melloch $\ddagger$}

* Department of Physics, Massachusetts Institute of Technology, Cambridge, Massachusetts 02139, USA

$\ddagger$ Department of Electrical Engineering, Purdue University, West Lafayette, Indiana 37907, USA

The unusual properties of two-dimensional electron systems that give rise to the quantum Hall effect have prompted the development of new microscopic models for electrical conduction ${ }^{1-6}$. The bulk properties of the quantum Hall effect have also been studied experimentally using a variety of probes including transport ${ }^{7,8}$, photoluminescence ${ }^{9,10}$, magnetization ${ }^{11}$ and capacitance ${ }^{12,13}$ measurements. However, the fact that two-dimensional electron systems typically exist some distance (about 1,000 $⿱$ ) beneath the surface of the host semiconductor has presented an important obstacle to more direct measurements of microscopic electronic structure in the quantum Hall regime. Here we introduce a cryogenic scanning-probe technique-'subsurface charge accumulation' imaging - that permits very high resolution examination of systems of mobile electrons inside materials. We use this technique to image directly the nanometre-scale electronic structures that exist in the quantum Hall regime.

The subsurface charge accumulation (SCA) probe measures the local accumulation of charge in a two-dimensional electron system (2DES) in response to an applied a.c. excitation. We use it to examine the quantum Hall system over areas as large as several micrometres, and with a spatial resolution of $\sim 900 \AA$. For magnetic fields near Hall plateaux, fine structures appear in the SCA images. These structures move and contort rapidly as the field is varied, even though they reappear on successive Hall plateaux.

In contrast to other techniques used to study a 2DES located beneath an insulating surface ${ }^{14,15}$, SCA imaging is an a.c. method ${ }^{16-19}$ and has significant advantages for the study of the quantum Hall effect over measurements which image the distribution of static charges. As samples contain static charges from both the surface and dopant layers underneath the surface, these regions can mask charging features in the two-dimensional layer ${ }^{15}$. SCA imaging, operating at frequencies of $\sim 100 \mathrm{kHz}$ (very low compared to other a.c. methods), is uniquely suited for studying the accumulation of mobile charges in the 2DES in magnetic fields.

SCA microscopy of the 2DES proceeds as follows. A sharp conducting tip is positioned $\sim 50 \AA$ above the sample surface and connected to a highly sensitive charge detector (Fig. 1). An a.c. excitation voltage is applied to the 2DES through an ohmic contact ( $2 \mathrm{~mm}$ away from the tip), and no other contacts are made to the sample. The excitation causes charge to flow in and out of the 2DES,

† Present address: Michigan State University, East Lansing, Michigan 48824, USA. in turn inducing charge to flow in and out of the tip. In scanning the tip and using synchronous lock-in detection of the induced charge, we obtain two images, one for charge accumulating in-phase $\left(Q_{\text {in }}\right)$ and the other $90^{\circ}$ (lagging) out-of-phase $\left(Q_{\text {out }}\right)$ with the excitation. The microscope also operates in other modes allowing determination of surface topography (tunnelling mode) and local accumulations of static charge (Kelvin probe mode) ${ }^{20}$.

As a test of our microscope for studying quantum Hall features, we first use it to locally perturb the 2DES. If large voltages $(>2 \mathrm{~V})$ are applied to the tip, static and immobile charge can be deposited on the exposed surface of the sample or in the donor layer between the surface and the 2DES, thereby locally altering the electron density in the 2DES. Figure 2 shows a series of six $Q_{\text {in }}$ (in-phase charging) SCA images centred on such a location. In these images, dark and bright represent respectively small and large $Q_{\text {in }}$ signals. We note the dark spot which appears as the field is raised to $1.5 \mathrm{~T}$ and grows for larger fields.

The origin of the dark spot can be understood by considering basic properties of the 2DES in the quantum Hall state. Any region containing a density which precisely fills an integer number of Landau levels is 'incompressible' and does not accumulate charge or conduct electricity. Such regions therefore appear dark in our images. At the centre of the images shown in Fig. 2, the electron density has a broad minimum (with the density reduced to about one-quarter of the bulk value). At the borders of the dark spot, the density is such that one Landau level is filled, preventing charge transport to the interior. Outside the dark spot, the density of electrons is high enough so that more than one level is still filled, and charge accumulates freely.

Incompressible regions such as this dark spot appear in some theories of the quantum Hall effect ${ }^{5,21,22}$ : they are introduced to explain the transitions between the quantum Hall plateaux. At

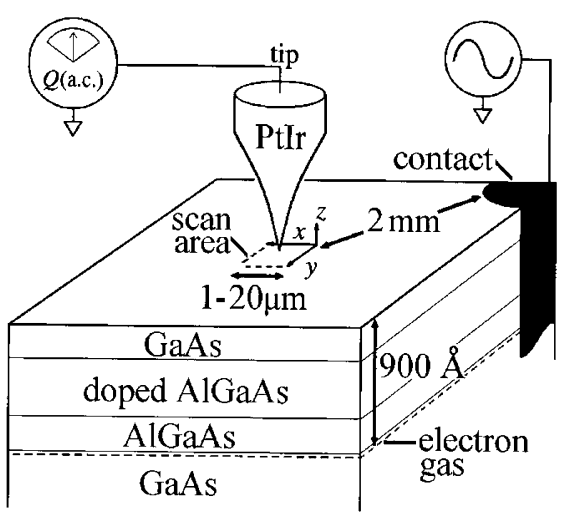

Figure 1 Diagram of the sample and measurement configuration. The twodimensional electron system (2DES) exists at the interface of $\mathrm{Al}_{0.3} \mathrm{Ga}_{0.7} \mathrm{As}$ and GaAs $900 \AA$ beneath the surface. The a.c. excitation applied to the 2DES causes this layer to charge and discharge due to the self-capacitance of the layer. A sharp metal tip is connected to a high electron mobility transistor (HEMT) with extremely high sensitivity to electrical charge ${ }^{30}$ (0.01 electrons $\left.\sqrt{\mathrm{H} z}\right)$ and scanned $50 \AA$ above the sample surface. SCA microscopy produces an image of charge accumulating in the 2DES by detecting charge induced on the tip due to charging in the layer. The spatial resolution is given approximately by the depth of the 2DES below the sample surface. The microscope operates with the sample immersed in liquid ${ }^{3} \mathrm{He}(T=300 \mathrm{mK})$ and has a $20-\mu \mathrm{m}$-long scan range. The static electron density in the 2DES is $3.0 \times 10^{11} \mathrm{~cm}^{-2}$ and the transport mobility is $450,000 \mathrm{~cm}^{2} \mathrm{~V}^{-1} \mathrm{~s}^{-1}$. The small amplitude of the $100 \mathrm{kHz}$ excitation applied to the 2DEG (1-4 mV) and the smallness of the capacitance between the tip and the 2DES (compared to the self-capacitance of the 2DES) ensure that the tip produces practically no perturbation of the $2 \mathrm{D}$ system. 
magnetic fields just below the quantum Hall plateaux, the theory predicts that the 2DES contains a system of incompressible droplets. As in the case of Fig. 2, as the field is increased, these droplets grow and begin to merge. Thus, entering the Hall plateau, the 2DES undergoes a percolation transition with the network of merged droplets impeding conduction across the sample, and thus producing a quantum Hall plateau ${ }^{1,4}$. However, in the absence of longrange potential fluctuations, theories do not require the existence of droplets at fixed positions ${ }^{23-25}$. The local charging observed in Fig. 2 displays sharp contrast, consistent with the concept of distinct insulating and conducting regions.

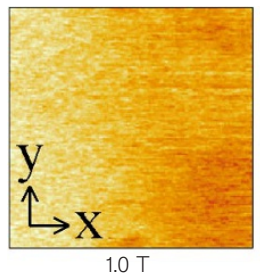

$1.0 \mathrm{~T}$

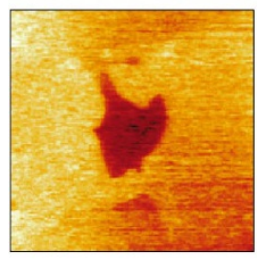

$3.0 \mathrm{~T}$
$1.5 \mathrm{~T}$

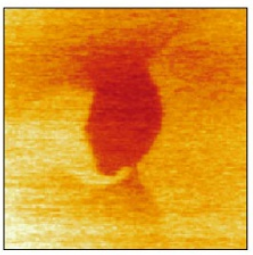

$3.8 \mathrm{~T}$
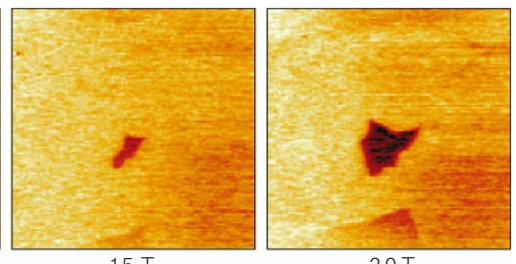

$2.0 \mathrm{~T}$

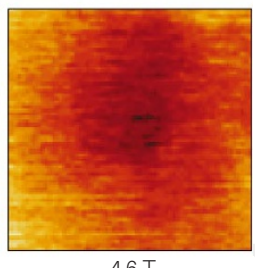

4.6 T

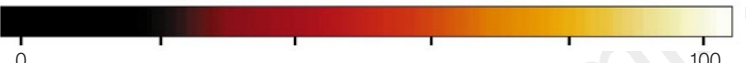

In-phase charge $\left(10^{1} 8 \mathrm{C} \mathrm{V}^{1}\right)$
Having shown that the microscope can resolve quantum Hall features, we now use it to examine an unperturbed system. In contrast to the perturbed region, the SCA images only develop features over narrow ranges in magnetic field around integer Landau level filling $(v)$ values. As the magnetic field is adjusted towards the centre of the Hall plateau, the charge accumulation seems to diminish roughly uniformly at all locations and detailed spatial patterns of charging appear.

Here we concentrate on the $v=4$ Hall plateau which is centred around $3.04 \mathrm{~T}$ in this sample. Images taken at fields of more than $0.15 \mathrm{~T}$ away from the centre of the Hall plateau show no features.

Figure 2 In-phase $\left(Q_{\text {in }}\right)$ SCA images of a region of perturbed electron density in the 2DES. Images $(2.7 \times 2.7 \mu \mathrm{m})$ are shown for various magnetic fields applied perpendicular to the $2 \mathrm{D}$ plane. Static charge measurements made in Kelvin probe mode show the 2D electron density has a shallow minimum at the centre of the images and increases to the bulk value away from the centre. For a magnetic field as high as 1.0T, the SCA image has few features. But at 1.5 T, the signal drops to a lower value when the tip is located near the centre, displaying a small feature (dark spot) which grows with increasing magnetic field. The density reduction at the spot centre can be explained by considering the magnetic field at which the black spot appears and by following its subsequent growth with field. In the unperturbed 2DES, the filling factor $v=2$ (one Landau level filled with both spinup and spin-down electrons) occurs at 6.1 T. The black spot appears at $1.5 \mathrm{~T}$, and grows monotonically as the field is raised. As this growth is observed to occur beyond the field for $v=3$ in the bulk, we conclude that the spot must first appear when the filling factor at the centre of the perturbed region is $v=2$. The spot emerges at one-quarter of the field required for $v=2$ in the bulk, suggesting that the density at the spot has been reduced to about one-quarter of the bulk density.
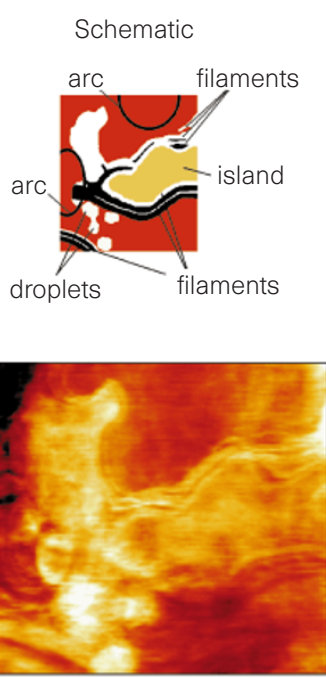

$3.000 \mathrm{~T}$

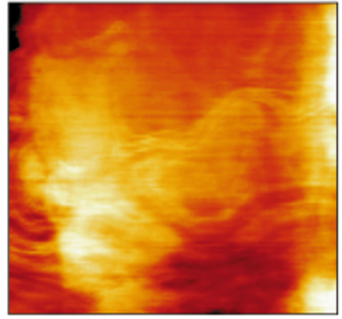

$2.990 \mathrm{~T}$

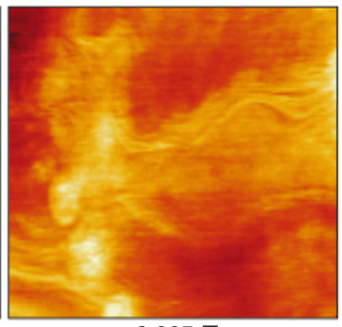

$3.005 \mathrm{~T}$

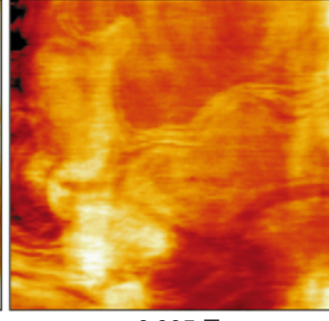

$2.995 \mathrm{~T}$

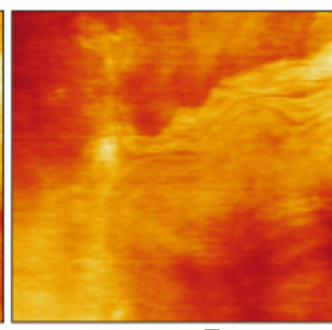

$3.010 \mathrm{~T}$

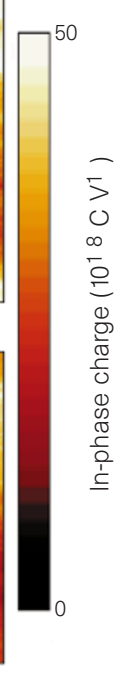

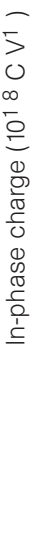

Figure 3 Sequence of five in-phase SCA images for an unperturbed region of the 2DES at $v \approx 4.05$. The images $(3.3 \times 3.3 \mu \mathrm{m})$ are for magnetic fields differing by $0.005 \mathrm{~T}$, and each image was obtained using $5 \mathrm{~h}$ of signal averaging. Kelvin probe measurements were used to find a region unperturbed by static charge fluctuations. The contact potential was measured with the microscope in Kelvin probe mode, and the tip bias (+0.80 V) was then set to eliminate d.c. fields between the tip and the 2DES. To enhance our ability to display small-scale features, a background plane has been subtracted from each image. This does not alter the images significantly nor create structural artefacts in the images. We note that, despite the great sensitivity of the structures to changes in magnetic field, the structures do not change at all with time. The expected spatial resolution of the probe is $900 \AA$, consistent with the smallest features displayed in the images. 'Filaments' (see schematic diagram at top left) of high or low charge accumulation appear in each of the images. In the 3.000 T image, these filaments are separated by $\sim 1,500 \AA$ and meander in unison for a distance of at least $2 \mu \mathrm{m}$ in the image. The filaments exist at the boundary between broader regions of high and low charge accumulation. Some filaments appear as broad dark bands that wind all the way across the image. Also, a grouping of high charge accumulating bulbous 'droplets' is clearly shown in the images at 3.000 Tand 3.005 T. The droplets shrink rapidly in size as the field strength is increased. We also note that 'arcs' or 'loops' appear in the images. The upper arc shown in the schematic exists in the image at $2.995 \mathrm{~T}$, and it appears to follow the circumference of a loop of diameter $\sim 1.5 \mu \mathrm{m}$. 
This indicates that spatial fluctuations in the charging (per volt of excitation amplitude) are less than $1 \times 10^{-18} \mathrm{CV}^{-1}$. For comparison, the dark region observed at $3.20 \mathrm{~T}$ in the perturbed area (Fig. 2) displayed a reduction of $50 \times 10^{-18} \mathrm{CV}^{-1}$. In contrast, clear and intricate charging features of amplitudes in the range of $20 \times 10^{-18} \mathrm{CV}^{-1}$ are observed for fields near the plateau centre. The features are extraordinarily sensitive to magnetic field, with little correlation between the images at fields differing by $0.020 \mathrm{~T}$ (or $0.7 \%)$.

In Fig. 3 we show a series of five images taken at magnetic fields spaced by $0.005 \mathrm{~T}$. The images reveal a startling amount of detail (see Fig. 3 legend). One may quickly ascertain that the features all indeed arise from charging structure within the two-dimensional layer resulting from the quantum Hall effect. There is no model for electronic structure in the donor layer or on the sample surface having such profound field sensitivity. Moreover, the structures are only seen when the magnetic field is near Hall plateaux. Last, features redevelop when the magnetic field is increased by precisely a factor of two, corresponding to the change from $v=4$ to $v=2$; the path followed by the filaments at 3.000 $\mathrm{T}$ (see schematic in Fig. 3) is once again followed by filaments only at precisely $6.000 \mathrm{~T}$ (near $v=2$ ). No known physical process would give rise to such periodicity other than the quantum Hall effect.

What is the origin of the contrast in the 2DES? The observed structure may arise from spatial variation in compressibility of the 2DES (that is, some regions of the 2DES can absorb more charge than others), or may be due to differences in local charging rates. It is possible to distinguish between these two mechanisms by comparing the contrast in the $Q_{\text {in }}$ and $Q_{\text {out }}$ images.

A simple approach to the 2DES is to consider it as an $R C$ system (where $R$ is the resistance of the layer, and $C$ is its self-capacitance).

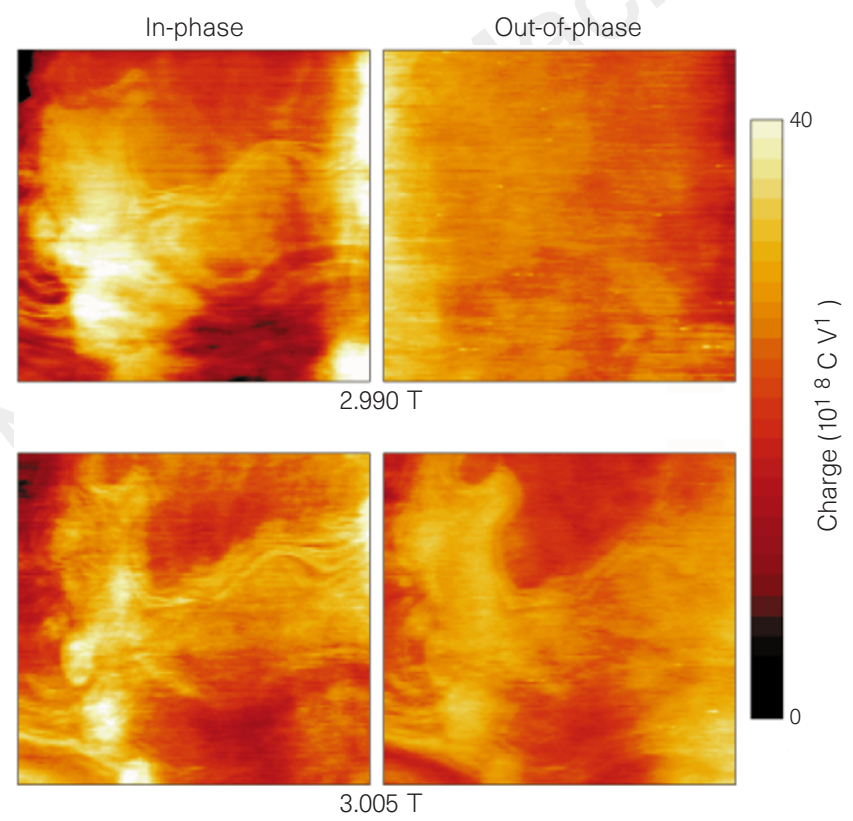

Figure $4 Q_{\text {in }}$ and $Q_{\text {out }}$ images at 2.990T (top row) and 3.005T (bottom row). We note that $Q_{\text {out }}$ is nearly featureless at 2.990 T while $Q_{\text {in }}$ displays detailed structure. Increasing the field by only 150 gauss to $3.005 \mathrm{~T}$ dramatically alters the phase of the image. The overall resistance of the sample increases as the field is raised from 2.990 to $3.005 \mathrm{~T}$, and the model for charging of the 2DES (see text) explains the observed phase shift. At 3.005T, the contrast seen in $Q_{\text {out }}$ always has the same sign as that in $Q_{\text {in. }}$. Based on a simple $R C$ charging model, it is possible to show that this observation is consistent with our conclusion that the structures in the images arise from variations in local compressibility and not from variation in local charging rates.
In our experiment, the resistivity of the sample varies as a function of magnetic field, and becomes extremely large at integer filling. At these fields, the roll-off frequency $f_{0}=1 /(2 \pi R C)$ may approach the excitation frequency $(100 \mathrm{kHz})$. As a result, in narrow intervals of field around integer fillings we detect both $Q_{\text {in }}$ and $Q_{\text {out }}$ components; that is, the signal has a non-zero phase shift. But at fields somewhat higher or lower than this interval, no detailed contrast is observed in $Q_{\text {out }}$ but much is seen in $Q_{\text {in }}$. In Fig. 4 we display this behaviour by showing $Q_{\text {in }}$ and $Q_{\text {out }}$ images for two different magnetic fields, with the higher field $(3.005 \mathrm{~T})$ closer to integer Landau level filling factor.

A more elaborate model treats the 2DES as a distributed $R C$ system with no unique roll-off frequency. Consider a region of size $L$, ranging from the size of the sample $L_{\max }$ (several millimetres) to the resolution limit given by $L_{\min }(900 \AA)$. Given a sheet of uniform resistivity in two dimensions, the effective resistance of this region does not depend on its size, but the self capacitance increases linearly with $L$. The roll-off frequency depends on the size $L$ of the region in question: $f_{0}(L)=1 /(2 \pi R L)$. Thus, $f_{0}(L)$ increases as $L$ diminishes. Therefore, when the roll-off frequency becomes of the order of the measurement frequency, the charging of smaller length scales still behaves as in the low-frequency limit, and features charge in-phase with their surroundings. Thus only compressibility features can be detected at small length scales.

Why is the contrast due to compressibility variation seen in such a narrow interval of magnetic fields? The simplest answer is that the features may exist only near integer fillings. Another possibility is that the contrast is enhanced near integer fillings because of the lack of screening by the 2DES. This enhancement will occur when the screening radius in the 2DES becomes of the order of the distance to the tip, that is, in a narrow interval around an integer filling.

We now consider what properties of the quantum Hall effect could give rise to the observed structures. From measurements of the sample capacitance versus magnetic field, we know that $v=4$ occurs at 3.040 $\mathrm{T}$ in the bulk. The droplets (see schematic in Fig. 3) seen at $3.000 \mathrm{~T}$ and $3.005 \mathrm{~T}$ can be explained as regions of higher electron density than their surroundings, which contain electrons in Landau levels $v>4$. As the magnetic field strength is increased, the higher Landau levels depopulate, and the droplets shrink in $\operatorname{size}^{26}$ and eventually disappear altogether.

At lower fields, the droplets are larger and at $2.995 \mathrm{~T}$ appear to overlap with a relatively bright 'island'. We thus surmise that the island is also a region of higher electron density where $v>4$. Filaments border this area and thus occur at positions where there is a density gradient. We note that we have never observed filaments which terminate: either they form closed loops or meander all the way across the images.

To understand the origin of filaments one has to explain clumping of the electron density in a 2DES where the main interaction between electrons is Coulomb repulsion. It is known that in some cases the exchange interaction between electrons can overcome the direct density-density interaction and change the effective interaction from repulsion to attraction. There are several models that use exchange and predict filamentary structures in the quantum Hall system. In particular, Chamon and $\mathrm{Wen}^{27}$ and MacDonald ${ }^{28}$ have described charge structures forming near the edges of a 2DES in the presence of a large density gradient. Recently, Koulakov, Fogler and Shklovskii ${ }^{29}$ considered a 2DES containing slightly greater than integer number of filled Landau levels, which is the same situation as Fig. 3. Using the Hartree-Fock method, Koulakov et al. found that the electrons in the higher level tended to form charge density waves; that is, a family of equally spaced filaments.

When the "island" (Fig. 3) is observed as a function of field, it is clear that unlike the "droplets", this region does not shrink but rather expands with increasing magnetic field. This at first seems inconsistent with the idea that the island contains electrons in a higher Landau level which depopulates as the field is raised. 
However, as the field increases, more dark filaments appear towards the centre of the island. In general, as the field is increased, the filaments widen into dark and bright bands moving outwards from the centre of the island. The images suggest that some regions of higher $v$ depopulate in a different fashion to that considered previously. In the island, the density of electrons in the higher Landau level is reduced by 'stretching' the area. The electron density in the island can be thought of as an accordion, and the spacing between bands of high electron density increases with increasing magnetic field.

We have shown that the SCA technique has a profound capability to look into systems that have been inaccessible to imaging with comparable resolution. Even though it is not at present possible to determine the exact origin of the structures, there is no question that they exist. The observed shapes, rapidly evolving in magnetic field, directly mirror the electronic structures in the highly correlated Hall fluid. The range of experimental conditions which produce such shapes, the importance of disorder, and the nature of the fractional Hall regime will be objects of further study.

Received 4 September; accepted 22 December 1997

1. Prange, R. E. \& Girvin, S. M. (eds) The Quantum Hall Effect 2nd edn (Springer, New York, 1990).

2. Das Sarma, S. \& Pinczuk, A. (eds) Perspectives in Quantum Hall Effects (Wiley Inter-Science, New York, 1997).

3. Laughlin, R. B. Quantized Hall conductivity in two dimensions. Phys. Rev. B 23, 5632-5634 (1981)

4. Halperin, B. I. Quantized Hall conductance, current-carrying edge states, and the existence of extended states in a two-dimensional disordered potential. Phys. Rev. B 25, 2185-2188 (1982).

Iordansky, S. V. On the conductivity of two dimensional electrons in a strong magnetic field. Solid State Commun. 43, 1-3 (1982).

6. Chalker, J. T. The quantum Hall effect: a sum rule. Surf. Sci. 142, 182-185 (1984).

. von Klitzing, K., Dorda, G. \& Pepper, M. New method for high-accuracy determination of the finestructure constant based on quantized Hall resistance. Phys. Rev. Lett. 45, 494-497 (1980).

8. Tsui, D. C., Stormer, H. L. \& Gossard, A. C. Zero-resistance state of two-dimensional electrons in a quantizing magnetic field. Phys. Rev. B 25, 1405-1407 (1982).

9. Goldberg, B. B. et al. Optical investigations of the integer and fractional quantum Hall effects: energy plateaus, intensity minima, and line splitting in band-gap emission. Phys. Rev. Lett. 65, 641-644 (1990).

10. Kukushkin, I. V. et al. Evidence of the triangular latice of crystallized electrons from time resolved luminescence. Phys. Rev. Lett. 72, 3594-3597 (1994).

11. Eisenstein, J. P. et al. Density of states and de Haas-van Alphen effect in two-dimensional electron systems. Phys. Rev. Lett. 55, 875-878 (1985).

12. Ashoori, R. C. \& Silsbee, R. H. The Landau level density of states as a function of Fermi energy in the two-dimensional electron gas. Solid State Commun. 81, 821-825 (1992).

13. Smith, T. P., Goldberg, B. B., Stiles, P. J. \& Heiblum, M. Direct measurement of the density of states of a two-dimensional electron gas. Phys. Rev. B 32, 2696-2699 (1985).

14. Eriksson, M. A., Beck, R. G., Topinka, M. \& Katine, J. A. Cryogenic scanning probe characterization of semiconductor nanostructures. Appl. Phys. Lett. 69, 671-673 (1996).

15. Yoo, M. J. et al. Scanning single-electron transistor microscopy: imaging individual charges. Science 276, 579-582 (1997)

16. Williams, C. C., Slinkman, J. \& Hough, W. P. Lateral dopant profiling with $200 \mathrm{~nm}$ resolution by scanning capacitance microscopy. Appl. Phys. Lett. 55, 1662-1664 (1989).

17. Kochanski, G. P. Nonlinear alternating-current tunneling microscopy. Phys. Rev. Lett. 62, 2285-2288 (1989).

18. Stranick, S. J. \& Weiss, P. S. A tunable microwave frequency alternating current scanning tunneling microscope. Rev. Sci. Instrum. 65, 918-921 (1994).

19. Bourgoin, J.-P., Johnson, M. B. \& Michel, B. Semiconductor characterization with the scanning surface harmonic microscope. Appl. Phys. Lett. 65, 2045-2047 (1994).

20. Shockley, W., Queisser, H. J. \& Hooper, W. W. Charges on oxidized silicon surfaces. Phys. Rev. Lett. 11, 489-490 (1963).

21. Efros, A. L. Non-linear screening and the background density of states of $2 \mathrm{DEG}$ in magnetic field. Solid State Commun. 67, 1019-1022 (1988)

22. Chklovskii, D. B. \& Lee, P. A. Transport properties between quantum Hall plateaus. Phys. Rev. B 48 18060-18078 (1993)

23. Trugman, S. A. Localization, percolation, and the quantum Hall effect. Phys. Rev. B 27, 7539-7546 (1983).

24. Pruisken, A. M. M. Universal singularities in the integral quantum Hall effect. Phys. Rev. Lett. 61, 1297-1300 (1988)

25. Huckenstein, B. Scaling theory of the integer quantum Hall effect. Rev. Mod. Phys. 67, 357-396 (1994).

26. McEuen, P. L. et al. Self-consistent addition spectrum of a Coulomb island in the quantum Hall regime. Phys. Rev. B 45, 11419-11422 (1992).

27. Chamon, C. d. C. \& Wen, X. G. Sharp and smooth boundaries of quantum Hall liquids. Phys. Rev. B 49, 8277-8241 (1994).

28. MacDonald, A. H., Yang, S. R. E. \& Johnson, M. D. Quantum dots in strong magnetic fields: stability criteria for the maximum density droplet. Aust. J. Phys. 46, 345-358 (1993).

29. Koulakov, A. A., Fogler, M. M. \& Shklovskii, B. I. Charge density wave in two-dimensional electron liquid in magnetic field. Phys. Rev. Lett. 76, 499-502 (1996).

30. Ashoori, R. C. et al. Single-electron capacitance spectroscopy of discrete quantum levels. Phys. Rev Lett. 68, 3088-3091 (1992).

Acknowledgements. We thank A. Cohen, E. Atmaca, M. Brodsky, H. B. Chan, A. Folch, S. Heemeyer A. Shytov, D. Silevitch and N. B. Zhitenev for technical help, and B. I. Halperin, H. F. Hess, R. B. Laughlin, P. A. Lee and B. I. Shklovskii for discussions. This work was supported by the Office of Naval Research, the Packard Foundation, JSEP, and the National Science Foundation DMR.

Correspondence and requests for materials should be addressed to R.C.A. (e-mail: ashoori@MIT.EDU)

\section{Highly mobile electrons and holes on isolated chains of the semiconducting polymer poly(phenylene vinylene)}

\author{
Romano J. O. M. Hoofman, Matthijs P. de Haas, \\ Laurens D. A. Siebbeles \& John M. Warman
}

IRI, Delft University of Technology, Mekelweg 15, 2629 JB Delft, The Netherlands

The nature of the charge carriers in 'conducting' polymers is of considerable interest at present ${ }^{1,2}$, largely on the basis of the technological potential of these materials for use as the semiconducting layer in field-effect transistors (FETs) and the emissive layer in light-emitting diodes $^{3}$ (LEDs). One of the main outstanding questions concerns the relative importance of intraversus inter-chain charge transfer in determining the overall rate of charge transport. Here we apply the pulse-radiolysis time-resolved microwave conductivity technique ${ }^{4}$ to dilute solutions of a soluble dialkoxy derivative of the semiconducting polymer poly(phenylene vinylene), PPV, by which means we determine the one-dimensional intra-chain mobilities of electrons and holes on isolated polymer chains free from inter-chain interactions. The values so obtained-0.5 and $0.2 \mathrm{~cm}^{2} \mathrm{~V}^{-1} \mathrm{~s}^{-1}$ respectively-are considerably larger than the mobilities measured previously for bulk PPV-based materials ${ }^{5-9}$. This suggests that considerable improvement in the performance characteristics (in particular switching time and maximum current) of organic FET and LED devices should be possible if material purity and structural order can be better controlled.

In this work, we used a dilute solution in benzene of poly(2methoxy-5-[2'-ethyl-hexyloxy]-phenylene vinylene), 'MEH-PPV'. The phenylene vinylene (PV) repeat-unit concentration, as determined by optical absorption, was $2 \times 10^{-3} \mathrm{M}$. This corresponds to a concentration of $2 \times 10^{-6} \mathrm{M}$ of polymer chains with an average length of $\sim 1,000 \mathrm{PV}$ units based on a relative molecular mass of $200,000 \pm 100,000$. We have found no evidence of aggregation occurring in the benzene solution of MEH-PPV used. Certain other dialkoxy-PPVs have been found to have varying tendencies to aggregate in solution, as evidenced by gel formation, the development of new adsorption and emission bands ${ }^{10,11}$, and in particular a very marked increase in the photoconductivity due to the occurrence of inter-chain charge transfer in aggregated systems ${ }^{11}$. We observed none of these effects for the present MEH-PPV solution, even after it had been standing in a refrigerator for several weeks.

Charge carriers are created uniformly throughout the solution by pulsed ionization using 5-ns pulses of 3-MeV electrons from a Van de Graaff accelerator; these electrons have a penetration depth $(15 \mathrm{~mm})$ much larger than the $3.5 \mathrm{~mm}$ thickness of the sample. Energy is initially absorbed from the beam mainly by the solvent, resulting in the formation of benzene radical cations ('holes'), $\mathrm{Bz}^{+}$, and excess electrons, $\mathrm{e}^{-}$. We calculated the concentration of electron-hole pairs formed in the pulse (using the known free ion yield of 0.053 per $100 \mathrm{eV}$ absorbed $\mathrm{d}^{12,13}$ and the accurately measured dose) to be $\sim 1 \times 10^{-7} \mathrm{M}$, that is, considerably less than the concentration of polymer chains in the solution.

Liquid benzene has an electron affinity ${ }^{14}$ of $\sim 0.1 \mathrm{eV}$, which is much lower than the $\sim 2.5 \mathrm{eV}$ estimated ${ }^{15}$ for PPV in the condensed phase. The excess electrons initially formed in the solvent would therefore be expected to react with the polymer chains in the solution according to:

$$
\mathrm{e}^{-}+\mathrm{PPV} \rightarrow \mathrm{PPV}^{-}
$$

